\title{
Zoom Case: um protótipo de case e software de apoio ao ensino e a aprendizagem de pessoas com baixa visão
}

\author{
Jonas Rocha Araújo, Luís Eduardo Anunciado Silva
}

\author{
Instituto Metrópole Digital - Universidade Federal do Rio Grande do Norte (UFRN) \\ Av. Senador Salgado Filho, 3000 - 59.078-970 - Natal - RN - Brasil \\ \{jonasra4, luis.eduardo.225\}@gmail.com
}

\begin{abstract}
Resumo. $O$ alto custo das tecnologias assistivas, que podem ampliar as habilidades funcionais de pessoas com deficiência, é um problema para que sejam utilizadas por indivíduos com baixa visão, em diversos contextos, dentre os quais o educacional. Diante disso, decidiu-se desenvolver um protótipo de baixo custo nomeado Zoom Case, para a inclusão de pessoas com baixa visão em todos os níveis da Educação. O Zoom Case é um dispositivo composto por uma capa para smartphone, fabricada por meio de impressão 3D, acoplado ao dispositivo móvel, que possibilitará a ampliação da imagem na tela em conjunto com um aplicativo que detêm diversas texturas sobre o texto para modificar o contraste e se adaptar a necessidade específica de cada usuário.
\end{abstract}

Endereço do vídeo: https://www.youtube.com/watch?v=dQqWglivjSQ

\section{Cenário de uso}

De acordo com a $10^{\mathrm{a}}$ classificação Estatística Internacional das Doenças e Problemas relacionados à Saúde (CID-10), a baixa visão ocorre quando a acuidade visual corrigida no melhor olho é menor do que 0,3 e maior ou igual a 0,05 ou seu campo visual é menor do que 20 graus (diâmetro) de campo visual no melhor olho, com a melhor correção óptica. Assim, a baixa visão ocorre quando a visão de um indivíduo estiver abaixo de $20 \%$, não podendo ser corrigida ou atenuada com o uso de óculos, cirurgia refrativa ou cirurgia de catarata. As principais causas da baixa visão são degeneração macular relacionada à idade, glaucoma e retinopatia diabética.

Segundo o Instituto Brasileiro de Geografia e Estatística (IBGE), por meio de dados do Censo 2010, existem mais de 6,5 milhões de pessoas com deficiência visual no Brasil, onde 6 milhões desse total são pessoas com baixa visão. Além disso, de acordo com a Organização Mundial da Saúde (OMS), no mundo, existem mais de 285 milhões de pessoas com deficiência visual, das quais 246 milhões são pessoas com baixa visão. Além disso, o número de pessoas com baixa visão irá aumentar como resultado do envelhecimento da população mundial.

Nesse contexto, a tecnologia da informação proporcionou o desenvolvimento de aparelhos especiais, como a lupa digital, que podem facilitar a vida de uma pessoa com baixa visão, possibilitando a leitura de impressos com auxílios ópticos ampliando a imagem lida consideravelmente, podendo se tornar um grande diferencial na vida escolar, acadêmica e profissional. Entretanto, por não serem desenvolvidos nacionalmente, estes produtos acabam por ter um custo de produção, manutenção e exportação elevados. Como exemplo, tem-se a lupa digital com um custo em torno de mil e quinhentos reais, tornando-se na maioria das 
vezes inacessível, fazendo com que uma parcela significativa das pessoas com baixa visão não tenham acesso a este recurso.

Sendo assim, pensando na inclusão em programas de educação da maioria das pessoas com baixa visão, desde a Educação Básica, passando pelo Ensino Técnico até a Educação Superior, desenvolveu-se o protótipo de software acoplado a uma case nominado Zoom Case, que possibilitará os mesmos resultados de aparelhos digitais a custos mais baixos.

\section{Desenvolvimento}

Para o desenvolvimento do projeto, visitou-se o Instituto de Educação e Reabilitação de Cegos do RN (IERC) em Natal/RN, um instituto sem fins lucrativos, reconhecido por sua utilidade pública municipal e estadual com registro no Conselho Nacional de Assistência Social. O IERC tem a finalidade de promover a habilitação, reabilitação e educação das pessoas com deficiências visuais. Durante a visita, percebeu-se diversos problemas relacionados à deficiência visual, dentre os quais destacou-se o alto custo da lupa digital, um dos dispositivos que eram mais utilizados em sala de aula para leitura de impressos, assim, na maioria das vezes, inacessível a algumas pessoas, restringindo a aprendizagem do aluno com baixa visão.

Pensando no problema apresentado, viu-se a necessidade de se criar uma tecnologia assistiva, como recurso para ampliar as habilidades funcionais de pessoas com deficiência visual (PcDV), de baixo custo, configurando-se como uma alternativa à lupa digital. Assim, durante a realização da pesquisa, objetivando custos baixos de produção, pensou-se em utilizar o hardware de um smartphone acoplado em uma estrutura universal que pudesse oferecer, junto a um aplicativo, as mesmas funcionalidades da lupa digital.

Além disso, foi realizada uma busca afim de encontrar produtos que tivessem características semelhantes em termos de acessibilidade. Foram encontradas algumas empresas que desenvolvem e fornecem produtos, como a ZooMax e a Freedom Scientific, que comercializam lupas eletrônicas para pessoas com baixa visão. Outra empresa é a Easy-Macro, que detêm um produto descrito como um banda elástica acoplada a uma lente, em que tal aparato apenas serve para auxiliar a captura de imagens pequenas. Entretanto, a solução não possui um aplicativo que pudesse auxiliar as pessoas com baixa visão, que necessitam de filtros monocromáticos para alcançar um contraste para tornar possível a normalização da sua leitura. Além disso, em seus aparatos físicos, não apresenta uma distância focal normalizada, o que pode causar uma pior experiência ao usuário sobre o dispositivo.

Para criação do aplicativo para smartphones que usam Android, um sistema operacional (SO) baseado no núcleo Linux e atualmente desenvolvido pela empresa de tecnologia Google, primeiramente, foi utilizado a plataforma Unity. Tal plataforma, desenvolvida pela Unity Technologies, é usada principalmente para desenvolver videogames e simulações para computadores, consoles e dispositivos móveis, e tem como base as linguagens de programação C \# (pronunciada "C Sharp") e JavaScript, que proporcionou a criação rápida e intuitiva do aplicativo.

Em seguida, foi iniciado a implementação do aplicativo para o Android Studio, que tem como base a linguagem de programação Java e fornece ferramentas direcionadas para 
VI Congresso Brasileiro de Informática na Educação (CBIE 2017)

Anais dos Workshops do VI Congresso Brasileiro de Informática na Educação (WCBIE 2017)

criação de aplicativos em todos os tipos de dispositivos Android, além de ser o ambiente de desenvolvimento integrado (IDE) oficial para a plataforma Android. Para realização do aplicativo teve-se como base o aplicativo Camera Filter ${ }^{1}$, de código-fonte aberto sob a licença Apache 2.0 e o filtro Blueorange ${ }^{2}$, criado com WebGL (Web Graphics Library), uma API em JavaScript baseada no OpenGL ES para aplicações web utilizada na computação gráfica

Por fim, para fabricação da estrutura ou case, foi visto a necessidade de utilização de impressão em 3D, um processo usado para criar um objeto tridimensional em que camadas de algum material por meio da modelagem feita do objeto em um computador. Logo após a impressão da case, foi acoplada uma lente macro que proporciona um ganho significativo de ampliação da câmera existente no smartphone. A modelagem da Zoom Case é mostrada na figura 1, 2 e 3. Em seguida, a figura 4 mostra o modelo impresso da Zoom Case acoplada a um smartphone.

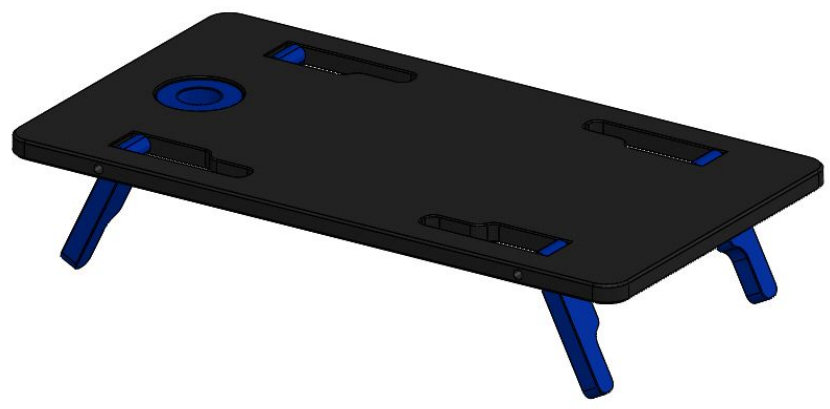

Figura 1: Visão frontal do modelo virtual da case.

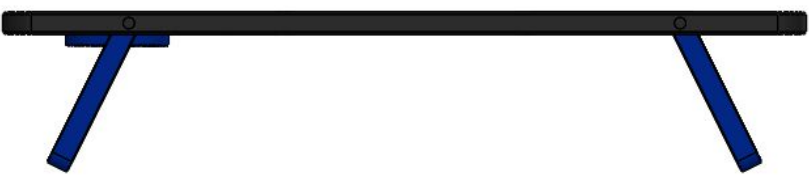

Figura 2: Visão lateral do modelo virtual da case.

\footnotetext{
${ }^{1}$ Github. CameraFilter: Realtime camera filters. Process frames by OpenGL shaders. Disponível em $<$ https://github.com/nekocode/CameraFilter>. Acessado em setembro de 2017.

2 Shadertoy. Blueorange. Disponível em <https://www.shadertoy.com/view/MsiGzr>. Acessado em setembro de 2017.
} 
VI Congresso Brasileiro de Informática na Educação (CBIE 2017)

Anais dos Workshops do VI Congresso Brasileiro de Informática na Educação (WCBIE 2017)

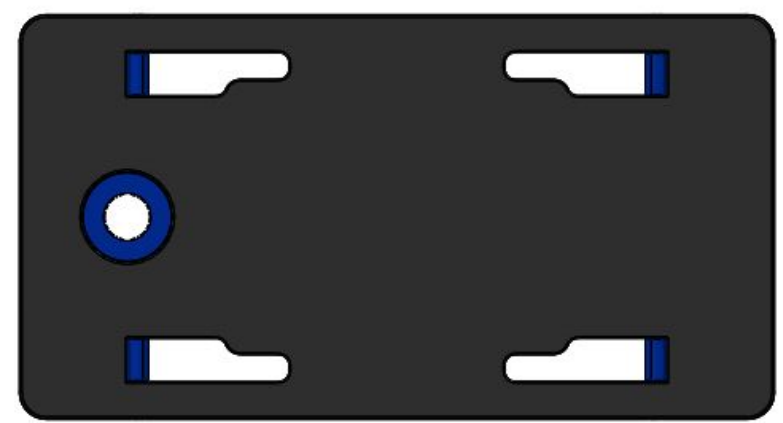

Figura 3: Visão superior do modelo virtual da case.

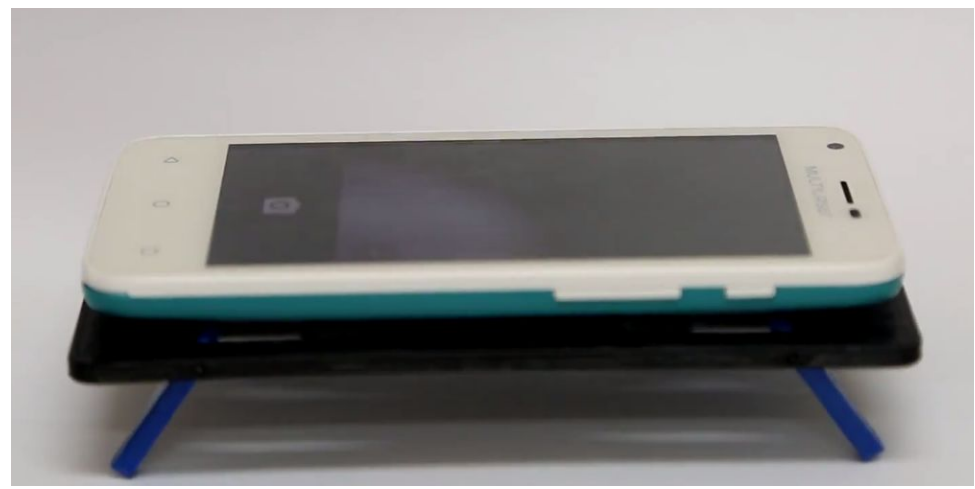

Figura 4: Visão lateral do modelo impresso da case acoplado a um smartphone.

\section{Apresentação do Software}

Em síntese, a aplicação é um filtro de câmera em tempo real, que tem seu processamento de quadros por meio do OpenGL (Open Graphics Library), uma API utilizada na computação gráfica, em que foi transferido o código do filtro Blue Orange utilizado em aplicações com WebGL para ser utilizado em aplicações com OpenGL ES.

Dessa forma, o protótipo do aplicativo foi desenvolvido para versões Android 2.2 ou superior e com a requisição aos usuários para concessão de permissão ao acesso a câmera. Assim, observando a figura 5 abaixo, onde há um diagrama de classes da aplicação, o funcionamento ocorre por meio da classe Main que chama a classe CameraRender, a principal classe do programa, sendo ela responsável pela inicialização da câmera, pela configuração de um mapa de filtros e pela criação de uma textura para visualização da dispositivo através da classe MyGLUtils. A CameraRender é inicializada sem texturas e ficará em loop até quando o usuário vier a selecionar algum dos filtros, em que a subclasse de 
VI Congresso Brasileiro de Informática na Educação (CBIE 2017)

Anais dos Workshops do VI Congresso Brasileiro de Informática na Educação (WCBIE 2017)

Filter respectiva ao filtro selecionado será chamado para criação de um buffer de renderização pela classe RenderBuffer e a construção de um sombreador que será renderizado em uma textura e logo após, visualizado na câmera. Por fim, na classe Main atráves de um método também é possível salvar imagens visualizadas na tela do aplicativo a qualquer momento, quando selecionado a opção do botão de salvar imagens no programa. Sendo esse o ciclo básico de operação do aplicativo até o fim de sua execução.

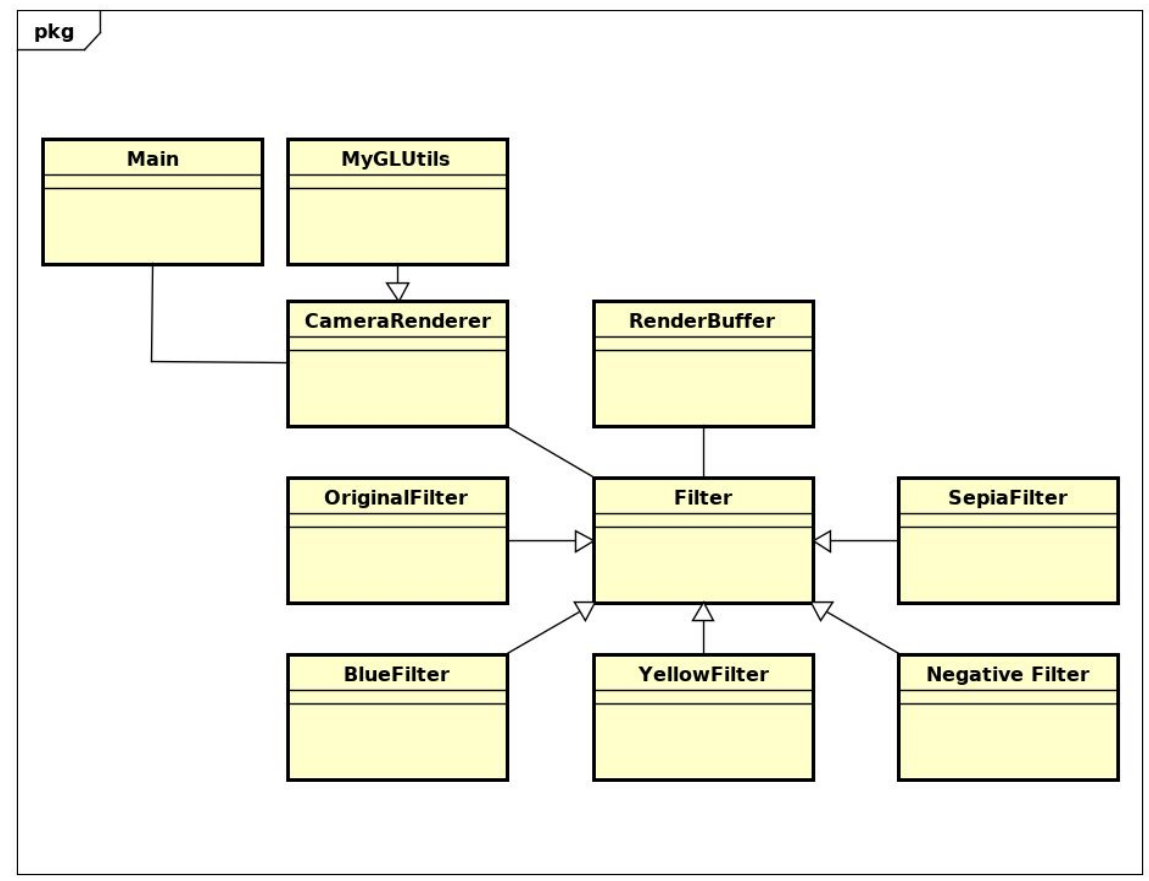

Figura 5: Diagrama de classes da aplicativo.

O protótipo da aplicação está sob a licença Apache 2.0 e tem como objetivo auxiliar nos processos de ensino e de aprendizagem das pessoas com baixa visão, em diferente etapas níveis e contextos da Educação. Com isso, o aplicativo da Zoom Case pode ser usado como uma ferramenta de auxílio empregada no aplicativo que utiliza a câmera inclusa no smartphone, podendo haver a ampliação significativa da imagem com o auxílio de uma lente macro inclusa na case, atráves de uma barra auxiliar lateral vista pelo usuário, ajustando o tamanho da letra de acordo com a necessidade de cada usuário do aplicativo.

Além disso, o aplicativo contará com uma aba lateral com diversas texturas, em que cada textura possa proporcionar ao usuário a forma mais agradável de se ler, dependendo da necessidade do usuário. Todo o layout do aplicativo foi desenvolvido para ser agradável, simples e intuitivo, permitindo que cada usuário possa usufruir de seus recursos com autonomia.

Por fim, o protótipo da Zoom Case é um dispositivo universal composto por uma capa integrada a uma lente macro, que acoplado a um smartphone, possibilita uma ampliação significativa da câmera inclusa no mesmo, além de um apoio que normaliza a distância focal. Ela será utilizada em conjunto com um aplicativo que detêm diversas texturas sobre o texto para aumentar o contraste e se adaptar a necessidade específica de cada usuário, além de armazenar em nuvem as imagens captadas pelo aplicativo. Sua fabricação será por meio de 
VI Congresso Brasileiro de Informática na Educação (CBIE 2017)

Anais dos Workshops do VI Congresso Brasileiro de Informática na Educação (WCBIE 2017)

impressão 3D, que possibilitará rapidez e, principalmente, um custo relativamente baixo, algo em torno de 30 reais, por produto. Além disso, pretende-se licenciar o projeto do protótipo conforme as licenças para open hardware.

\subsection{Funcionamento}

Apesar de estar no início deste projeto, o processo de desenvolvimento da case universal já se encontra em fase avançada, pois sua modelagem é rápida, pela estrutura não apresentar muitos detalhes. Entretanto o processo de desenvolvimento do aplicativo se encontra em fase bastante inicial, pois a construção de todo aplicativo foi feita em uma plataforma de rápida concepção, para um protótipo base. Logo após, o aplicativo foi migrado para outra plataforma que proporciona estabilidade do software. Assim, no momento, embora a implementação das texturas já tenha sido concluída, ainda há a necessidade da execução do zoom digital pelo aplicativo, para que possa proporcionar um aumento ainda maior da imagem, se restringindo ao tamanho da tela do smartphone.

Para baixar a aplicação acesse: https://github.com/cruxiu/app-zoom-case/raw/master/app/build/outputs/apk/app-zoom-case.a pk. O conceito das telas do aplicativo da Zoom Case é mostrada na figura 6, 7, 8, 9 e 10, em que cada figura contém uma textura diferente que se adequa a necessidade específica de cada usuário.

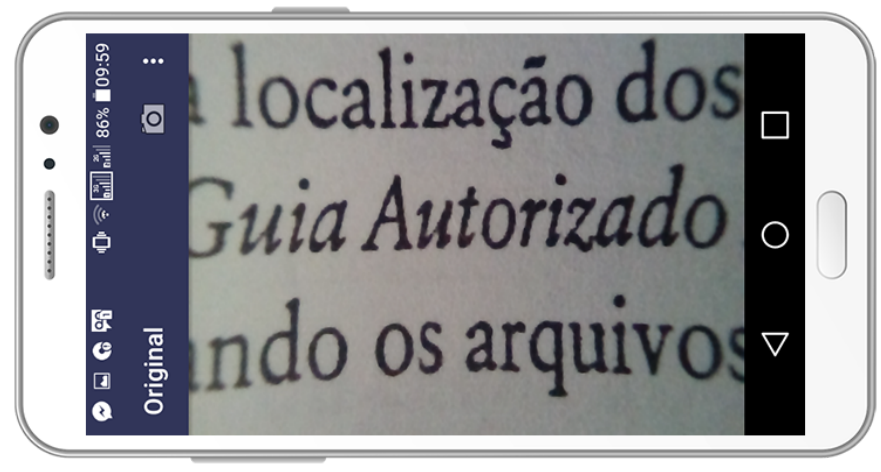

Figura 6: Tela principal do aplicativo Zoom Case (Sem textura).

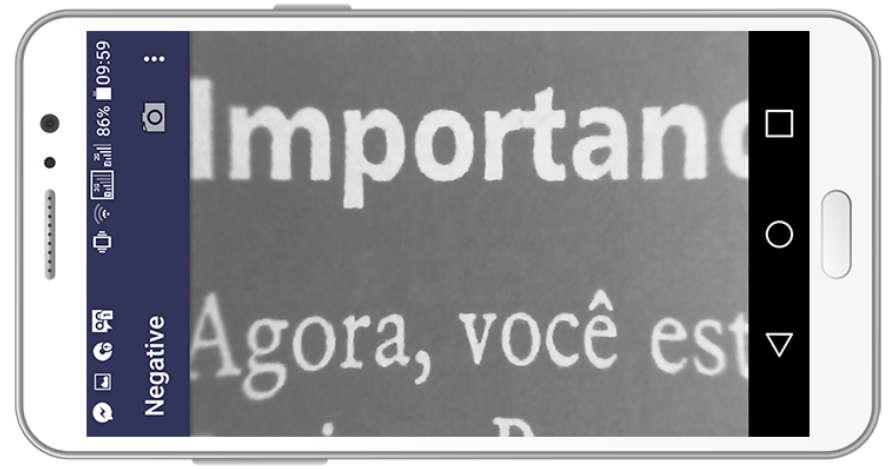

Figura 7: Tela principal do aplicativo Zoom Case (Com textura negativa). 
VI Congresso Brasileiro de Informática na Educação (CBIE 2017)

Anais dos Workshops do VI Congresso Brasileiro de Informática na Educação (WCBIE 2017)

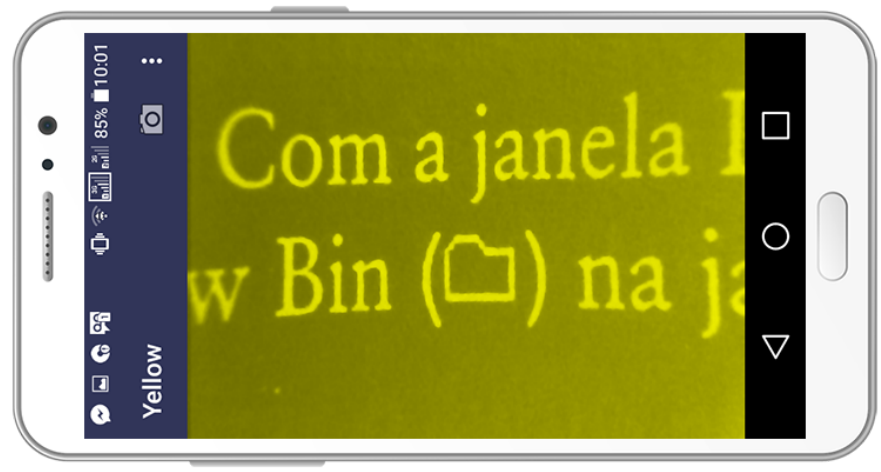

Figura 8: Tela principal do aplicativo Zoom Case (Com textura amarela).

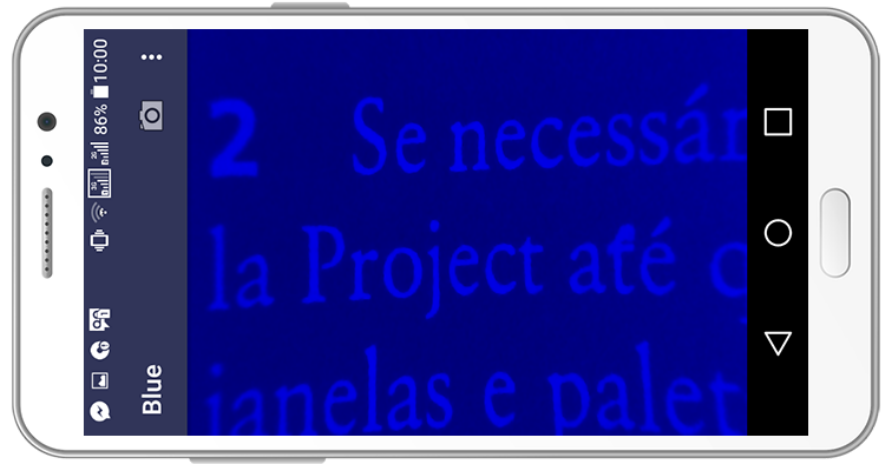

Figura 9: Tela principal do aplicativo Zoom Case (Com textura azul).

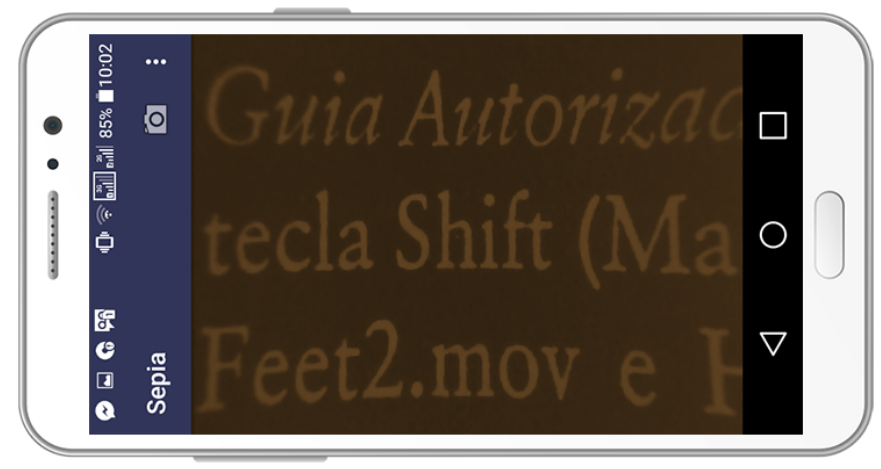

Figura 10: Tela principal do aplicativo Zoom Case (Com textura sépia).

\section{Considerações finais}

O desenvolvimento deste protótipo é direcionado a pessoas com baixa visão, sendo 
um recurso que amplia as habilidades funcionais dos usuários que a utilizarem. Todavia, ainda há a necessidade de testes de forma experimental, para verificar se há a adaptação de pessoas ao protótipo, além de empregar as modificações necessárias para o bom uso por todos que precisarem .

As funcionalidades do aplicativo ainda estão em fase de teste, onde já está disponível ao usuário diversas texturas sobre o texto. Entretanto ainda busca-se implementar a execução do zoom digital pelo programa, para que possa ampliar ainda mais a imagem, além da criação de novos modelos de case que se adaptem melhor a todos os usuários. Visamos assim, a melhoria dos recursos incluídos no aplicativo, melhorando o desempenho na execução e confortabilidade do usuário, para proporcionar a todos independência de ensino e aprendizagem a custos mais baixos.

Com o amadurecimento e consolidação do protótipo, pretende-se realizar testes em contextos reais de ensino e aprendizagem de PcDV. Com isso, espera-se o desenvolvimento de trabalhos futuros que apontem para usos pedagógicos da Zoom Case para pessoas com baixa visão. Essa solução de baixo custo pode contribuir não apenas para facilitar a vida estudantil e social dessas pessoas, mas para tornar algumas práticas de leitura possíveis e acessíveis a alguns toques na tela de um dispositivo móvel como o smartphone.

\section{Referências}

Apache License. Disponível em $<$ https://www.apache.org/licenses/LICENSE-2.0 $>$. Acessado em setembro de 2017.

Astah - Software Design Tools for Agile teams with UML, ER Diagram, Flowchart, Mindmap and More I Astah.net. Disponível em $<$ http://astah.net $>$. Acessado em setembro de 2017.

Cook, A. M. e Polgar, J. M. (2007) Cook and Hussey's Assistive Technologies: Principles and Practice, Mosby.

Easy-Macro: Macro lens for iPhone, Android, or any mobile device. Disponível em $<$ https://www.easy-macro.com/>. Acessado em setembro de 2017.

Github. CameraFilter: Realtime camera filters. Process frames by OpenGL shaders. Disponível em $<$ https://github.com/nekocode/CameraFilter $>$. Acessado em setembro de 2017.

IBGE Censo. Censo Demográfico 2010. Disponível em $<$ http://www.ibge.gov.br/home/estatistica/populacao/censo2010/default.shtm $>$. Acessado em junho de 2017.

Shadertoy. Blueorange. Disponível em <https://www.shadertoy.com/view/MslGzr>. Acessado em setembro de 2017.

World Health Organization. Global Data on Visual Impairments 2010. Disponível em $<$ http://www.who.int/blindness/GLOBALDATAFINALforweb.pdf $>$. Acessado em junho de 2017.

World Health Organization. ICD-10, International Classification of Diseases. Disponível em

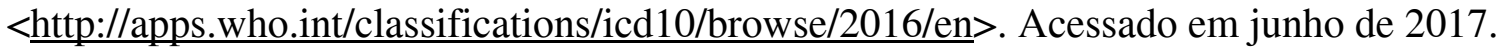

